\title{
APPLICATION OF THE ALTERNATING DIRECTION IMPLICIT METHOD FOR NUMERICAL SOLUTION OF THE DUAL PHASE LAG EQUATION
}

\author{
MARIUSz Ciesielski \\ Czestochowa University of Technology, Institute of Computer and Information Sciences, Częstochowa, Poland \\ e-mail: mariusz.ciesielski@icis.pcz.pl
}

\begin{abstract}
The problem discussed in the paper concerns the numerical modeling of thermal processes proceeding in micro-scale described using the Dual Phase Lag Model (DPLM) in which the relaxation and thermalization time appear. The cylindrical domain of a thin metal film subjected to a strong laser pulse beam is considered. The laser action is taken into account by the introduction of an internal heat source in the energy equation. At the stage of numerical modeling, the Control Volume Method is used and adapted to resolve the hyperbolic partial differential equation. In particular, the Alternating Direction Implicit (ADI) method for DPLM is presented and discussed. The examples of computations are also presented.
\end{abstract}

Keywords: micro-scale heat transfer, dual phase lag model, control volume method, alternating direction implicit method, numerical simulation

\section{Introduction}

Thermal processes proceeding in the microscale are characterized, as a rule, by an extremely short duration, extreme temperature gradients and very small geometrical dimensions of the domain considered. It is a reason that typical mathematical models basing on the macroscopic Fourier-type equations are not suitable for the analysis of this type problems. In the recent years, the problem of heat transfer through domains subjected to an strong external heat source (e.g. an ultrafast laser pulse) has been of vital importance in microtechnology applications, and it is a reason that the problems connected with fast heating of solids has become a very active research area (Tzou, 2015; Zhang, 2007; Chen et al., 2004).

From the mathematical point of view, nowadays there exist different models describing the mechanism of the process discussed. In this group, the microscopic two-temperature parabolic or hyperbolic models (belonging to a group of continuum models) should be mentioned (Chen and Beraun, 2001; Kaba and Dai, 2005; Lin and Zhigilei, 2008; Majchrzak, 2012; Majchrzak and Dziatkiewicz, 2015). The two-step parabolic and hyperbolic models involve two energy equations determining the thermal processes in the electron gas and the metal lattice. The coupling factor combining these equations is introduced. Depending on the variant of the model, parabolic or hyperbolic PDEs are considered. Assuming certain simplifications, the two-temperature model can be transformed into a single equation containing the second order time derivative and higher order mixed derivative in both time and space (known as the dual phase lag model (DPLM)). In this equation, two positive constants $\tau_{q}, \tau_{T}$ appear and they correspond to the relaxation time, which is the mean time for electrons to change their energy states and the thermalization time, which is the mean time required for electrons and lattice to reach equilibrium (Orlande et al., 1995).

The Cattaneo-Vernotte and the dual phase lag models belong also to the group of continuum ones. They result from the generalization of the well-known Fourier law. To take into account the finite velocity of a thermal wave the lag time between the heat flux and temperature gradient 
has been introduced (Cattaneo, 1958). The Cattaneo-Vernotte hyperbolic equation (CVE) can be treated as a certain microscale heat transfer model, but for this purpose is rarely used. The model discussed often finds application in the case of bioheat transfer problems, e,g. (Ciesielski et al., 2016). In fact, according to literature, e.g. (Mitra et al., 1995) the lag time (relaxation time) for processed meat is of the order of several seconds.

Introduction of two lag times in the generalized form of the Fourier law (relaxation and thermalization ones) leads, after relatively simple mathematical manipulations, to the dual phase lag equation. At present, in literature one can find big number of analytical and (first of all) numerical solutions of various thermal problems described by this model. The majority of the solutions presented in the literature concerns the 1D problems. Such an assumption is often fully acceptable. For example, considering the laser pulse interactions with thin metal films it is reasonable to treat the interactions as a one-dimensional heat transfer process (Chen and Beraun, 2001). In this paper, the axially-symmetrical problem is analyzed.

Most of the works in this field concerns direct problems. Homogeneous and also heterogeneous domains are considered. The problem of the single layer heating was discussed, among others, by Tang and Araki (1999), Kaba and Dai (2005), Mochnacki and Ciesielski (2012), Majchrzak and Turchan (2016). In the subject of non-homogeneous micro-domains, one can mention the paper presented by Dai and Nassar (2000), in which the heat transfer in a double layered goldchromium film is analyzed, and the papers prepared by Majchrzak et al. (2009a,b) concerning a multi-layered film subjected to ultrafast laser heating.

Both in the case of the CVE and DPLE, the typical boundary conditions appearing in heat transfer problems should be modified in a adequate way.

In literature, one can find works devoted to sensitivity of the transient temperature field in microdomains with respect to the dual phase lag model parameters (Majchrzak, and Mochnacki, 2014). The issue of the inverse problems was also developed, e.g. by Mochnacki and Paruch (2013), Dziatkiewicz et al. (2014), Mochnacki and Ciesielski (2015).

A next group of microscale heat transfer models is based on the Boltzmann transport equation (BTE). It is a conservation equation where the conserved quantity is the number of particles (Tian and Yang, 2008). The general form of BTE is rather complex, but it can be modified to analyze special tasks, for instance systems created by phonons, electrons, etc. In this field, deserving special attention is repeatedly cited paper presented by Escobar et al. (2006). One can also mention the work by Belhayat-Piasecka and Korczak (2016) in which the microscale heat transport was analyzed using the interval lattice Boltzmann method.

Microscale heat transfer processes can be also considered using the molecular approaches (Smith and Norris, 2003; Theodosiou and Saravanos, 2007; Chen et al., 2007; Liu and Tsai, 2009).

\section{Governing equations}

Let us consider the diffusion equation in the domain $\Omega$

$$
(r, z) \in \Omega \quad c \frac{\partial T(r, z, t)}{\partial t}=-\nabla \cdot \mathbf{q}(r, z, t)+Q(r, z, t)
$$

where $c=c(T)$ is the volumetric specific heat, $\mathbf{q}(r, z, t)$ is the heat flux vector, $Q(r, z, t)$ is the capacity of internal heat sources, $r, z, t$ are the geometrical co-ordinates and time.

The value of heat flux is determined by Tzou's dual-phase-lag theory (Tzou, 2015), as the generalization of the Fourier law, in particular

$$
\mathbf{q}\left(r, z, t+\tau_{q}\right)=-\lambda \nabla T\left(r, z, t+\tau_{T}\right)
$$


where $\tau_{q}$ is called the relaxation time, while $\tau_{T}$ is the thermalization time, $\lambda=\lambda(T)$ is the thermal conductivity, $\nabla T(r, z, t)$ is the temperature gradient. For $\tau_{T}=0$, this model leads to the Cattaneo-Vernotte equation, while for $\tau_{T}=0$ and $\tau_{q}=0$ it corresponds to the Fourier law.

The Taylor series expansions of equation (2.2) is the following

$$
\mathbf{q}(r, z, t)+\tau_{q} \frac{\partial \mathbf{q}(r, z, t)}{\partial t}=-\lambda\left[\nabla T(r, z, t)+\tau_{T} \frac{\partial \nabla T(r, z, t)}{\partial t}\right]
$$

Introducing formula (2.3) into equation (2.1) one obtains

$$
\begin{aligned}
& c\left[\frac{\partial T(r, z, t)}{\partial t}+\tau_{q} \frac{\partial^{2} T(r, z, t)}{\partial t^{2}}\right]=\nabla \cdot[\lambda \nabla T(r, z, t)]+\tau_{T} \frac{\partial \nabla \cdot[\lambda \nabla T(r, z, t)]}{\partial t} \\
& \quad+Q(r, z, t)+\tau_{q} \frac{\partial Q(r, z, t)}{\partial t}
\end{aligned}
$$

In the case of the axially-symmetrical task discussed in this work, the component $\nabla \cdot[\lambda \nabla T(r, z, t)]$ is the following

$$
\nabla \cdot[\lambda \nabla T(r, z, t)]=\frac{1}{r} \frac{\partial}{\partial r}\left[r \lambda \frac{\partial T(r, z, t)}{\partial r}\right]+\frac{\partial}{\partial z}\left[\lambda \frac{\partial T(r, z, t)}{\partial z}\right]
$$

It should be pointed out that the boundary conditions (which appear in the typical Fourier heat conduction models) for the DPL should be transformed to the form

$$
(r, z) \in \Gamma: \quad q_{b}(r, z, t)+\tau_{q} \frac{\partial q_{b}(r, z, t)}{\partial t}=-\lambda\left[\mathbf{n} \cdot \nabla T(r, z, t)+\tau_{T} \frac{\partial[\mathbf{n} \cdot \nabla T(r, z, t)]}{\partial t}\right]
$$

In Fig. 1, the domain considered (limited by the planes $z=0, z=Z$ and surface $r=R$ ) is shown.

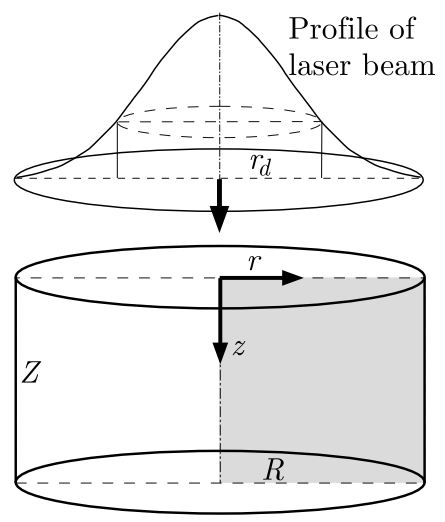

Fig. 1. Cylindrical micro-domain

The effects of femtosecond laser pulse irradiation on the upper surface limiting the system causes that the energy is delivered to metal and its absorption occurs. The internal heat source $Q(r, z, t)$ generated inside metal is related with action of the laser beam (Chen and Beraun, 2001)

$$
Q(r, z, t)=\sqrt{\frac{\beta}{\pi}} \frac{1-R_{f}}{t_{p} \delta} I_{0} \exp \left(-\frac{z}{\delta}\right) \exp \left(-\frac{r^{2}}{r_{d}^{2}}\right) \exp \left(-\beta\left(\frac{t-2 t_{p}}{t_{p}}\right)^{2}\right)=I_{\Omega}(r, z) I_{t}(t)
$$

where

$$
I_{\Omega}(r, z)=I_{0} \frac{1-R_{f}}{\delta} \exp \left(-\frac{r^{2}}{r_{d}^{2}}\right) \exp \left(-\frac{z}{\delta}\right) \quad I_{t}(t)=\frac{\sqrt{\beta}}{t_{p} \sqrt{\pi}} \exp \left(-\beta\left(\frac{t-2 t_{p}}{t_{p}}\right)^{2}\right)
$$


and $I_{0}$ is laser intensity, $R_{f}$ is reflectivity of the irradiated surface, $\delta$ is optical penetration depth, $r_{d}$ is characteristic radius of Gaussian laser beam, $\beta=4 \ln 2$ and $t_{p}$ is characteristic time of the laser pulse. Here, it is assumed that the total time of the laser action beam on the surface is equal to $4 t_{p}$.

So, action of the laser beam is taken into account by introduction of the internal heat source $Q(r, z, t)$. At the same time the dimensions $Z$ and $R$ are large enough that on the appropriate boundaries adiabatic conditions $q_{b}(r, z, t)=0$ can be assumed. In the case of the problem considered (see: Eq. (2.6)) one has

$$
(r, z) \in \Gamma: \quad-\lambda\left[\mathbf{n} \cdot \nabla T(r, z, t)+\tau_{T} \frac{\partial[\mathbf{n} \cdot \nabla T(r, z, t)]}{\partial t}\right]=0
$$

The initial conditions (the initial temperature of domain $T_{0}(r, z)$ and the initial heating rate $v_{0}(r, z)$ are also given

$$
t=0: \quad T(r, z, 0)=\left.T_{0}(r, z) \quad \frac{\partial T(r, z, t)}{\partial t}\right|_{t=0}=v_{0}(r, z)
$$

\section{Numerical solution using the Control Volume Method}

To solve the problem presented in the previous Section, the control volume method (CVM) is used. This method constitutes a very effective tool for numerical modeling of heat transfer processes described by the Fourier-type equations. In the case of numerical simulation of microscale heat transfer and the models based on the DPL equation, this method has so far been applied only to the numerical solution using an 'explicit' scheme (Mochnacki and Ciesielski, 2015).

The first stage of the method application is the division of the domain considered into small cells (known as the control volumes CV). In this work, the shape of control volumes is regular one (it corresponds to the rings of a rectangular cross-section). The more complex discretization using e.g. the Voronoi polygons can be also taken into account (Ciesielski and Mochnacki, 2014).

In Fig. 2, the domain discretization is presented, while in Fig. 3 the selected internal and boundary (top) control volumes are shown.
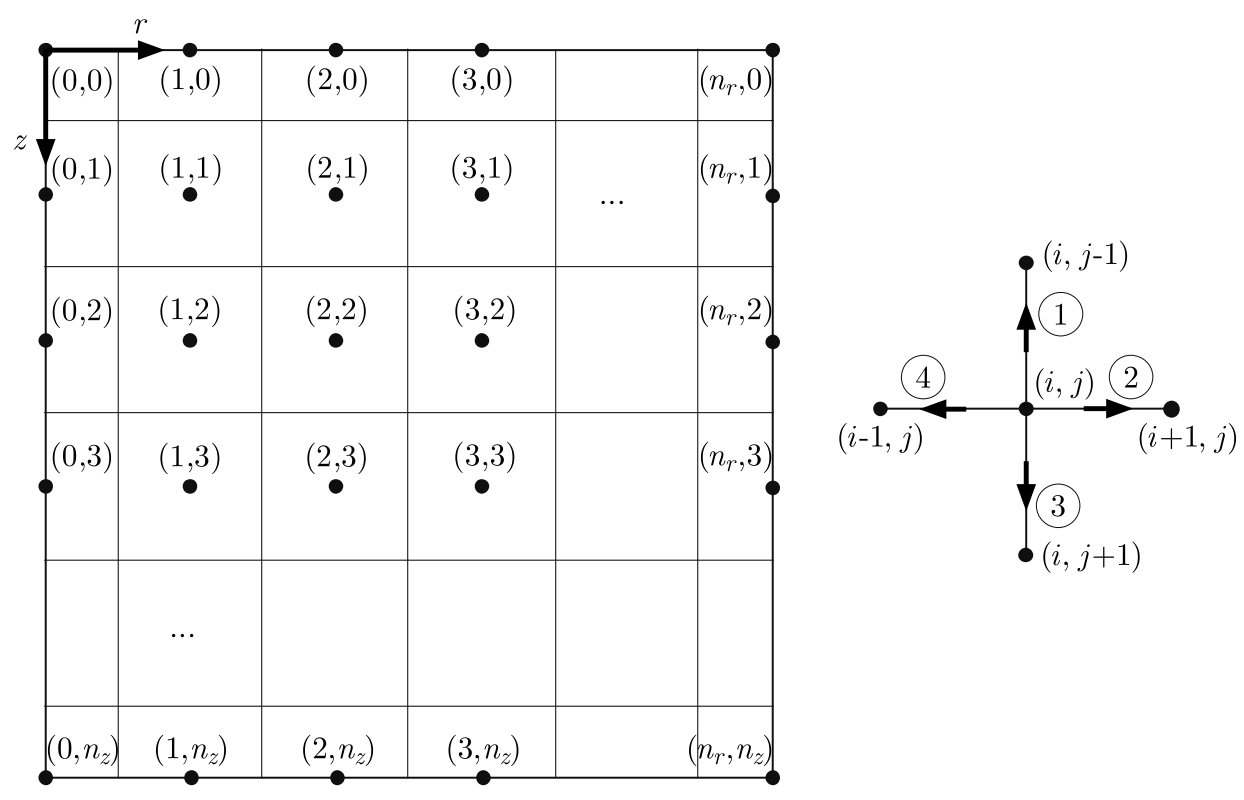

Fig. 2. Discretization of the domain 

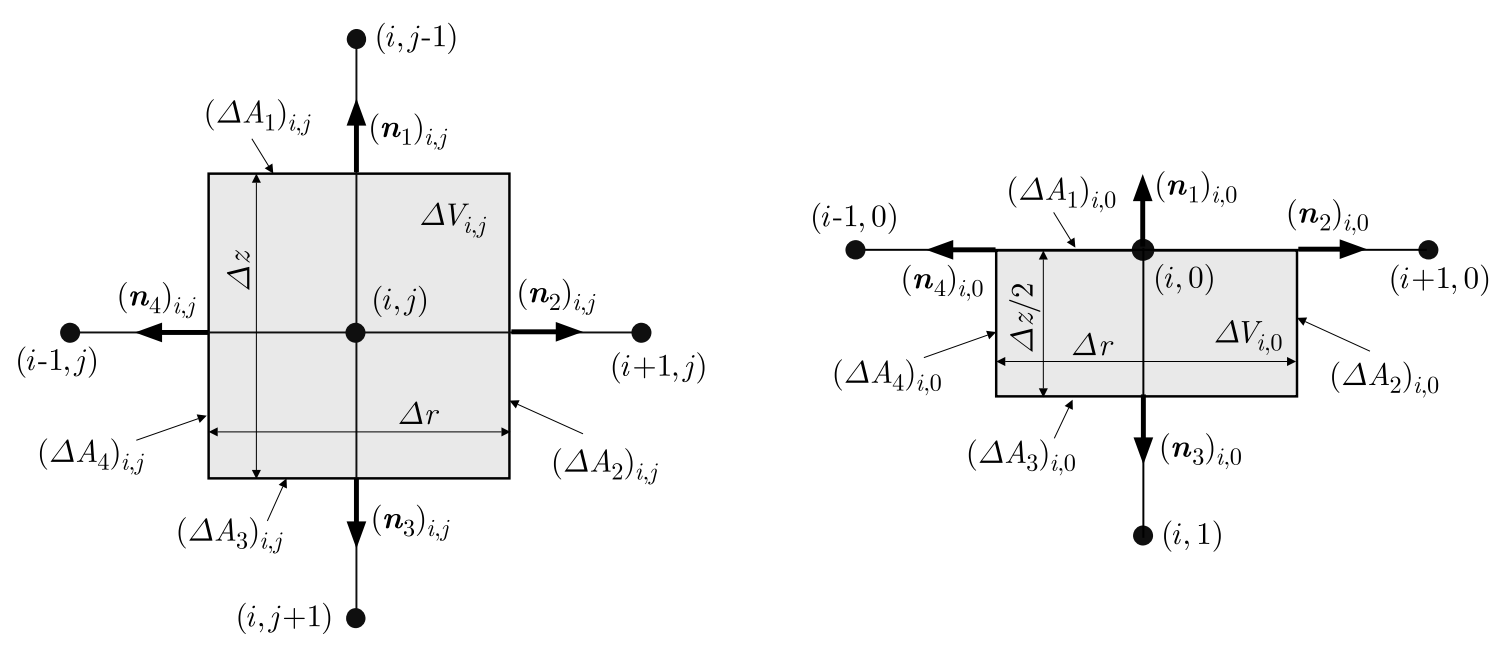

Fig. 3. The internal and boundary control volumes

On the basis of simple geometrical considerations, one can determine values of the successive volumes $\Delta V_{i, j}$ and surfaces $\left(\Delta A_{k}\right)_{i, j}$ limiting $\Delta V_{i, j}$ in each $k$-direction. Numerical modelling of transient problems requires introduction of the time grid, too: $0=t^{0}<t^{1}<\ldots<t^{f}<\ldots<t^{F}$, $t^{f}=f \Delta t$.

The aim of the CVM is to find the transient temperature field at the set of control volumes. The thermal capacities are concentrated at the elements, whereas the thermal resistances are concentrated on the sectors connecting nodes of the control volumes. The average temperatures in all control volumes can be found on the basis of energy balances for the successive volumes. The energy balances corresponding to the heat exchange between the analyzed control volume and adjacent control volumes results from integration of the energy equation with respect to volume and time.

\subsection{Integration of the energy equation with respect to volume}

Integration of Eq. (2.4) over the control volume $\Omega_{i, j}$ leads to

$$
\begin{array}{rl}
\int_{\Omega_{i, j}} & c\left(\frac{\partial T(r, z, t)}{\partial t}+\tau_{q} \frac{\partial^{2} T(r, z, t)}{\partial t^{2}}\right) d \Omega \\
& =\int_{\Omega_{i, j}}\left(\nabla \cdot[\lambda \nabla T(r, z, t)]+\tau_{T} \frac{\partial \nabla \cdot[\lambda \nabla T(r, z, t)]}{\partial t}\right) d \Omega+\int_{\Omega_{i, j}}\left(Q(r, z, t)+\tau_{q} \frac{\partial Q(r, z, t)}{\partial t}\right)^{(3.1)} d \Omega
\end{array}
$$

The integral occurring on the left-hand side of equation (3.1) can be approximated in the form

$$
\begin{aligned}
& \int_{\Omega_{i, j}} c\left(\frac{\partial T(r, z, t)}{\partial t}+\tau_{q} \frac{\partial^{2} T(r, z, t)}{\partial t^{2}}\right) d \Omega \\
& \cong c_{i, j}\left(\left.\frac{\partial T(r, z, t)}{\partial t}\right|_{\substack{r=r_{i} \\
z=z_{j}}}+\left.\tau_{q} \frac{\partial^{2} T(r, z, t)}{\partial t^{2}}\right|_{\substack{r=r_{i} \\
z=z_{j}}}\right) \Delta V_{i, j}=c_{i, j}\left(\frac{d T_{i, j}}{d t}+\tau_{q} \frac{d^{2} T_{i, j}}{d t^{2}}\right) \Delta V_{i, j}
\end{aligned}
$$

where $T_{i, j}=T\left(r_{i}, z_{j}, t\right)$, while $c_{i, j}=c\left(T_{i, j}\right)$ is the integral mean of thermal capacity in the volume $\Omega_{i, j}$. In a similar way, the numerical approximation of the source term in Eq. (3.1) can be found

$$
\int_{\Omega_{i, j}}\left(Q(r, z, t)+\tau_{q} \frac{\partial Q(r, z, t)}{\partial t}\right) d \Omega \cong\left(Q_{i, j}+\tau_{q} \frac{d Q_{i, j}}{d t}\right) \Delta V_{i, j}
$$


where $Q_{i, j}$ is determined as the integral mean of the heat source in the volume $\Omega_{i, j}$

$$
\begin{aligned}
Q_{i, j} & \equiv Q_{i, j}(t) \cong \frac{1}{\Delta V_{i, j}} \int_{\Omega_{i, j}} Q(r, z, t) d \Omega=I_{t}(t) \frac{1}{\Delta V_{i, j}} \int_{\Omega_{i, j}} I_{\Omega}(r, z) d \Omega \\
& =I_{t}(t) \frac{I_{0} \pi\left(1-R_{f}\right) r_{d}^{2}}{\Delta V_{i, j}}\left[\exp \left(-\frac{\bar{r}_{1}^{2}}{r_{d}^{2}}\right)-\exp \left(-\frac{\bar{r}_{2}^{2}}{r_{d}^{2}}\right)\right]\left[\exp \left(-\frac{\bar{z}_{1}}{\delta}\right)-\exp \left(-\frac{\bar{z}_{2}}{\delta}\right)\right]
\end{aligned}
$$

and $\bar{r}_{1}, \bar{r}_{2}, \bar{z}_{1}, \bar{z}_{2}$ are the limits of the control volume $\Omega_{i, j}=\left\{\left(r_{i}, z_{i}\right) \mid \bar{r}_{1} \leqslant r_{i} \leqslant \bar{r}_{2}, \bar{z}_{1} \leqslant z_{i} \leqslant \bar{z}_{2}\right\}$. In the case of a more complex form of the function $Q$, one can compute $Q_{i, j}(t)=Q\left(r_{i}, z_{j}, t\right)$, but this estimation is less accurate.

Applying the divergence theorem to the term determining heat conduction (right hand side of Eq. (3.1)) between the volume $\Omega_{i, j}$ bounded by the surfaces $\Delta A_{i, j}$ and its neighbourhoods, one obtains

$$
\begin{aligned}
\int_{\Omega_{i, j}} & \left(\nabla \cdot[\lambda \nabla T(r, z, t)]+\tau_{T} \frac{\partial \nabla \cdot[\lambda \nabla T(r, z, t)]}{\partial t}\right) d \Omega \\
= & \int_{\Omega_{i, j}} \nabla \cdot \lambda\left(\nabla T(r, z, t)+\tau_{T} \frac{\partial \nabla T(r, z, t)}{\partial t}\right) d \Omega \\
= & \int_{A_{i, j}}\left[\mathbf{n} \cdot \lambda\left(\nabla T(r, z, t)+\tau_{T} \frac{\partial \nabla T(r, z, t)}{\partial t}\right)\right] d A
\end{aligned}
$$

and then this term can be written in the form

$$
\begin{aligned}
\int_{A_{i, j}} & {\left[\mathbf{n} \cdot \lambda\left(\nabla T(r, z, t)+\tau_{T} \frac{\partial \nabla T(r, z, t)}{\partial t}\right)\right] d A } \\
& =\sum_{k=1}^{4} \int_{\left(A_{k}\right)_{i, j}}\left[\left(\mathbf{n}_{k}\right)_{i, j} \cdot\left(\lambda_{k}\right)_{i, j}\left(\left.\nabla T(r, z, t)\right|_{k}+\left.\tau_{T} \frac{\partial \nabla T(r, z, t)}{\partial t}\right|_{k}\right)_{i, j}\right] d A_{k} \\
& \cong \sum_{k=1}^{4}\left(\mathbf{n}_{k}\right)_{i, j} \cdot\left(\lambda_{k}\right)_{i, j}\left(\left.\nabla T(r, z, t)\right|_{k}+\left.\tau_{T} \frac{\partial \nabla T(r, z, t)}{\partial t}\right|_{k}\right)_{i, j}\left(\Delta A_{k}\right)_{i, j}=\sum_{k=1}^{4}\left(q_{k}\right)_{i, j}\left(\Delta A_{k}\right)_{i, j}
\end{aligned}
$$

where $\left(q_{k}\right)_{i, j}$ is approximated by the following finite differences (taking into account also the adiabatic boundary conditions (2.9) on the boundary surfaces)

$$
\begin{aligned}
& \left(q_{1}\right)_{i, j}= \begin{cases}\left(\lambda_{1}\right)_{i, j}\left[\frac{T_{i, j-1}-T_{i, j}}{\Delta z}+\tau_{T} \frac{d}{d t}\left(\frac{T_{i, j-1}-T_{i, j}}{\Delta z}\right)\right] & \text { if } \quad j>0 \\
0 & \text { if } \quad j=0\end{cases} \\
& \left(q_{2}\right)_{i, j}= \begin{cases}\left(\lambda_{2}\right)_{i, j}\left[\frac{T_{i+1, j}-T_{i, j}}{\Delta r}+\tau_{T} \frac{d}{d t}\left(\frac{T_{i+1, j}-T_{i, j}}{\Delta r}\right)\right] & \text { if } i<n_{r} \\
0 & \text { if } \quad i=n_{r}\end{cases} \\
& \left(q_{3}\right)_{i, j}=\left\{\begin{array}{lll}
\left(\lambda_{3}\right)_{i, j}\left[\frac{T_{i, j+1}-T_{i, j}}{\Delta z}+\tau_{T} \frac{d}{d t}\left(\frac{T_{i, j+1}-T_{i, j}}{\Delta z}\right)\right] & \text { if } j<n_{z} \\
0 & \text { if } \quad j=n_{z}
\end{array}\right. \\
& \left(q_{4}\right)_{i, j}=\left\{\begin{array}{lll}
\left(\lambda_{4}\right)_{i, j}\left[\frac{T_{i-1, j}-T_{i, j}}{\Delta r}+\tau_{T} \frac{d}{d t}\left(\frac{T_{i-1, j}-T_{i, j}}{\Delta r}\right)\right] & \text { if } \quad i>0 \\
0 & \text { if } \quad i=0
\end{array}\right.
\end{aligned}
$$


and $\left(\lambda_{k}\right)_{i, j}$ are harmonic mean thermal conductivities between two central points of adjoining control volumes

$$
\begin{aligned}
\left(\lambda_{1}\right)_{i, j} & =\frac{2 \lambda_{i, j} \lambda_{i, j-1}}{\lambda_{i, j}+\lambda_{i, j-1}} & \left(\lambda_{2}\right)_{i, j} & =\frac{2 \lambda_{i, j} \lambda_{i+1, j}}{\lambda_{i, j}+\lambda_{i+1, j}} \\
\left(\lambda_{3}\right)_{i, j} & =\frac{2 \lambda_{i, j} \lambda_{i, j+1}}{\lambda_{i, j}+\lambda_{i, j+1}} & \left(\lambda_{4}\right)_{i, j} & =\frac{2 \lambda_{i, j} \lambda_{i-1, j}}{\lambda_{i, j}+\lambda_{i-1, j}}
\end{aligned}
$$

and next, the thermal resistances are defined as follows

$$
\left(R_{1}\right)_{i, j}=\frac{\Delta z}{\left(\lambda_{1}\right)_{i, j}} \quad\left(R_{2}\right)_{i, j}=\frac{\Delta r}{\left(\lambda_{2}\right)_{i, j}} \quad\left(R_{3}\right)_{i, j}=\frac{\Delta z}{\left(\lambda_{3}\right)_{i, j}} \quad\left(R_{4}\right)_{i, j}=\frac{\Delta r}{\left(\lambda_{4}\right)_{i, j}}
$$

Then, Eq. (3.6) takes the form

$$
\int_{A_{i, j}}\left[\mathbf{n} \cdot \lambda\left(\nabla T(r, z, t)+\tau_{T} \frac{\partial \nabla T(r, z, t)}{\partial t}\right)\right] d A \cong \sum_{k=1}^{4} \frac{\left(\theta_{k}\right)_{i, j}}{\left(R_{k}\right)_{i, j}}\left(\Delta A_{k}\right)_{i, j}
$$

where

$$
\begin{aligned}
& \left(\theta_{1}\right)_{i, j}=\left.\left(T_{i, j-1}-T_{i, j}+\tau_{T} \frac{d\left(T_{i, j-1}-T_{i, j}\right)}{d t}\right)\right|^{\text {if } j>0} \\
& \left(\theta_{2}\right)_{i, j}=\left.\left(T_{i+1, j}-T_{i, j}+\tau_{T} \frac{d\left(T_{i+1, j}-T_{i, j}\right)}{d t}\right)\right|^{\text {if } i<n_{r}} \\
& \left(\theta_{3}\right)_{i, j}=\left.\left(T_{i, j+1}-T_{i, j}+\tau_{T} \frac{d\left(T_{i, j+1}-T_{i, j}\right)}{d t}\right)\right|^{\text {if } j<n_{z}} \\
& \left(\theta_{4}\right)_{i, j}=\left.\left(T_{i-1, j}-T_{i, j}+\tau_{T} \frac{d\left(T_{i-1, j}-T_{i, j}\right)}{d t}\right)\right|^{\text {if } i>0}
\end{aligned}
$$

while the notation expression ||$^{\text {if condition }}$ introduced above, means

$$
\text { expression }\left.\right|^{\text {if condition }}= \begin{cases}\text { expression } & \text { if condition }=\text { true } \\ 0 & \text { otherwise }\end{cases}
$$

After the introduction of all discrete terms into equation (3.1), one obtains

$$
c_{i, j}\left(\frac{d T_{i, j}}{d t}+\tau_{q} \frac{d^{2} T_{i, j}}{d t^{2}}\right) \Delta V_{i, j}=\sum_{k=1}^{4} \frac{\left(\theta_{k}\right)_{i, j}}{\left(R_{k}\right)_{i, j}}\left(\Delta A_{k}\right)_{i, j}+\left(Q_{i, j}+\tau_{q} \frac{d Q_{i, j}}{d t}\right) \Delta V_{i, j}
$$

or

$$
c_{i, j}\left(\frac{d T_{i, j}}{d t}+\tau_{q} \frac{d^{2} T_{i, j}}{d t^{2}}\right)=\sum_{k=1}^{4} \frac{\left(\theta_{k}\right)_{i, j}}{\left(R_{k}\right)_{i, j}}\left(\Phi_{k}\right)_{i, j}+Q_{i, j}+\tau_{q} \frac{d Q_{i, j}}{d t}
$$

where $\left(\Phi_{k}\right)_{i, j}=\left(\Delta A_{k}\right)_{i, j} / \Delta V_{i, j}$. 


\subsection{Integration of the equation with respect to time}

The second stage of CVM is integration of equation (3.17) with respect to time. The same effect can be obtained introducing the approximation of time derivatives occurring in (3.17) by appropriate finite differences.

The idea of the ADI method is to split the time step $\Delta t=t^{f+1}-t^{f}$ into two half-steps and apply two different finite difference schemes for each half time step. In the first half time step, a simple implicit scheme for directions $\left(d_{1}, d_{2}\right)$ is used and simultaneously an explicit scheme for directions $\left(d_{3}, d_{4}\right)$ is applied. Next, in the second half time step, the difference schemas are written by reversing the directions of the explicit and implicit schemes. The notation ' $\left(d_{1}, d_{2}\right)-\left(d_{3}, d_{4}\right)$ ', where the indexes $d_{i}$ indicate directions of the neighbouring CV (see Fig. 1), is introduced.

For passing: $t^{f} \rightarrow t^{f+0.5} \rightarrow t^{f+1}, f=1, \ldots, F$, and using the variant of ADI: $(1,2)-(3,4)$, the following differential schemas are proposed

$$
\begin{aligned}
& c_{i, j}^{f}\left(\frac{T_{i, j}^{f+0.5}-T_{i, j}^{f}}{0.5 \Delta t}+\tau_{q} \frac{T_{i, j}^{f+0.5}-2 T_{i, j}^{f}+T_{i, j}^{f-0.5}}{(0.5 \Delta t)^{2}}\right) \\
& \quad=\sum_{k=1,2} \frac{\left(\theta_{k}\right)_{i, j}^{f+0.5}}{\left(R_{k}\right)_{i, j}^{f}}\left(\Phi_{k}\right)_{i, j}+\sum_{k=3,4} \frac{\left(\theta_{k}\right)_{i, j}^{f}}{\left(R_{k}\right)_{i, j}^{f}}\left(\Phi_{k}\right)_{i, j}+Q_{i, j}^{f+0.5}+\tau_{q} \frac{Q_{i, j}^{f+0.5}-Q_{i, j}^{f}}{0.5 \Delta t}
\end{aligned}
$$

and

$$
\begin{aligned}
& c_{i, j}^{f+0.5}\left(\frac{T_{i, j}^{f+1}-T_{i, j}^{f+0.5}}{0.5 \Delta t}+\tau_{q} \frac{T_{i, j}^{f+1}-2 T_{i, j}^{f+0.5}+T_{i, j}^{f}}{(0.5 \Delta t)^{2}}\right) \\
& \quad=\sum_{k=1,2} \frac{\left(\theta_{k}\right)_{i, j}^{f+0.5}}{\left(R_{k}\right)_{i, j}^{f+0.5}}\left(\Phi_{k}\right)_{i, j}+\sum_{k=3,4} \frac{\left(\theta_{k}\right)_{i, j}^{f+1}}{\left(R_{k}\right)_{i, j}^{f+0.5}}\left(\Phi_{k}\right)_{i, j}+Q_{i, j}^{f+1}+\tau_{q} \frac{Q_{i, j}^{f+1}-Q_{i, j}^{f+0.5}}{0.5 \Delta t}
\end{aligned}
$$

and $\left(\theta_{k}\right)_{i, j}^{s}$ for $s \in\{f, f+0.5, f+1\}$ for this method are approximated in the following way

$$
\begin{aligned}
& \left(\theta_{1}\right)_{i, j}^{s}=\left[T_{i, j-1}^{s}-T_{i, j}^{s}+\tau_{T}\left(\frac{\left.\left.T_{i, j-1}^{s}-T_{i, j-1}^{s-0.5}-\frac{T_{i, j}^{s}-T_{i, j}^{s-0.5}}{0.5 \Delta t}\right)\right]\left.\right|^{\text {if } j>0}}{0.5 \Delta t}\right)\right. \\
& \left(\theta_{2}\right)_{i, j}^{s}=\left.\left[T_{i+1, j}^{s}-T_{i, j}^{s}+\tau_{T}\left(\frac{T_{i+1, j}^{s}-T_{i+1, j}^{s-0.5}}{0.5 \Delta t}-\frac{T_{i, j}^{s}-T_{i, j}^{s-0.5}}{0.5 \Delta t}\right)\right]\right|^{\text {if } i<n_{r}} \\
& \left(\theta_{3}\right)_{i, j}^{s}=\left.\left[T_{i, j+1}^{s}-T_{i, j}^{s}+\tau_{T}\left(\frac{T_{i, j+1}^{s}-T_{i, j+1}^{s-0.5}}{0.5 \Delta t}-\frac{T_{i, j}^{s}-T_{i, j}^{s-0.5}}{0.5 \Delta t}\right)\right]\right|^{\text {if } j<n_{z}} \\
& \left(\theta_{4}\right)_{i, j}^{s}=\left.\left[T_{i-1, j}^{s}-T_{i, j}^{s}+\tau_{T}\left(\frac{T_{i-1, j}^{s}-T_{i-1, j}^{s-0.5}}{0.5 \Delta t}-\frac{T_{i, j}^{s}-T_{i, j}^{s-0.5}}{0.5 \Delta t}\right)\right]\right|^{\text {if } i>0}
\end{aligned}
$$

After transformations, the first system of equations (3.18) can be written in the final form as

$$
\left(A_{0}^{\prime}\right)_{i, j}^{f} T_{i, j}^{f+0.5}+\left.\left(A_{1}^{\prime}\right)_{i, j}^{f} T_{i, j-1}^{f+0.5}\right|^{\text {if } j>0}+\left.\left(A_{2}^{\prime}\right)_{i, j}^{f} T_{i+1, j}^{f+0.5}\right|^{\text {if } i<n_{r}}=\left(D^{\prime}\right)_{i, j}^{f}
$$

where 


$$
\begin{aligned}
& \left(A_{k}^{\prime}\right)_{i, j}^{f}=-\left(1+2 \mu_{T}\right) \frac{\left(\Phi_{k}\right)_{i, j}}{\left(R_{k}\right)_{i, j}^{f}} \quad k=1,2 \\
& \left(A_{0}^{\prime}\right)_{i, j}^{f}=2 c_{i, j}^{f} \frac{1+2 \mu_{q}}{\Delta t}-\left(\left.\left(A_{1}^{\prime}\right)_{i, j}^{f}\right|^{\text {if } j>0}+\left.\left(A_{2}^{\prime}\right)_{i, j}^{f}\right|^{\text {if } i<n_{r}}\right) \\
& \left(D^{\prime}\right)_{i, j}^{f}=\left.2 \mu_{T}\left(T_{i, j}^{f}-T_{i, j-1}^{f}\right) \frac{\left(\Phi_{1}\right)_{i, j}}{\left(R_{1}\right)_{i, j}^{f}}\right|^{\text {if } j>0}+\left.2 \mu_{T}\left(T_{i, j}^{f}-T_{i+1, j}^{f}\right) \frac{\left(\Phi_{2}\right)_{i, j}}{\left(R_{2}\right)_{i, j}^{f}}\right|^{\text {if } i<n_{r}} \\
& +\left.\left[-\left(1+2 \mu_{T}\right)\left(T_{i, j}^{f}-T_{i, j+1}^{f}\right)+2 \mu_{T}\left(T_{i, j}^{f-0.5}-T_{i, j+1}^{f-0.5}\right)\right] \frac{\left(\Phi_{3}\right)_{i, j}}{\left(R_{3}\right)_{i, j}^{f}}\right|^{\text {if } j<n_{z}} \\
& +\left.\left[-\left(1+2 \mu_{T}\right)\left(T_{i, j}^{f}-T_{i-1, j}^{f}\right)+2 \mu_{T}\left(T_{i, j}^{f-0.5}-T_{i-1, j}^{f-0.5}\right)\right] \frac{\left(\Phi_{4}\right)_{i, j}}{\left(R_{4}\right)_{i, j}^{f}}\right|^{\text {if } i>0} \\
& +2 c_{i, j}^{f} \frac{\left(1+4 \mu_{q}\right) T_{i, j}^{f}-2 \mu_{q} T_{i, j}^{f-0.5}}{\Delta t}+\left(1+2 \mu_{q}\right) Q_{i, j}^{f+0.5}-2 \mu_{q} Q_{i, j}^{f}
\end{aligned}
$$

while the second system of equations (3.19) - in the following form

$$
\left(A_{0}^{\prime \prime}\right)_{i, j}^{f+0.5} T_{i, j}^{f+1}+\left.\left(A_{3}^{\prime \prime}\right)_{i, j}^{f+0.5} T_{i, j+1}^{f+1}\right|^{\text {if } j<n_{z}}+\left.\left(A_{4}^{\prime \prime}\right)_{i, j}^{f+0.5} T_{i-1, j}^{f+1}\right|^{\text {if } i>0}=\left(D^{\prime \prime}\right)_{i, j}^{f+0.5}
$$

where

$$
\begin{aligned}
& \left(A_{k}^{\prime \prime}\right)_{i, j}^{f+0.5}=-\left(1+2 \mu_{T}\right) \frac{\left(\Phi_{k}\right)_{i, j}}{\left(R_{k}\right)_{i, j}^{f+0.5}} \quad k=3,4 \\
& \left(A_{0}^{\prime \prime}\right)_{i, j}^{f+0.5}=2 c_{i, j}^{f+0.5} \frac{1+2 \mu_{q}}{\Delta t}-\left(\left.\left(A_{3}^{\prime \prime}\right)_{i, j}^{f+0.5}\right|^{\text {if } j<n_{z}}+\left.\left(A_{4}^{\prime \prime}\right)_{i, j}^{f+0.5}\right|^{\text {if } i>0}\right) \\
& \left(D^{\prime \prime}\right)_{i, j}^{f+0.5}=\left.2 \mu_{T}\left(T_{i, j}^{f+0.5}-T_{i, j+1}^{f+0.5}\right) \frac{\left(\Phi_{3}\right)_{i, j}}{\left(R_{3}\right)_{i, j}^{f+0.5}}\right|^{\text {if } j<n_{z}}+\left.2 \mu_{T}\left(T_{i, j}^{f+0.5}-T_{i-1, j}^{f+0.5}\right) \frac{\left(\Phi_{4}\right)_{i, j}}{\left(R_{4}\right)_{i, j}^{f+0.5}}\right|^{\text {if } i>0} \\
& +\left.\left[-\left(1+2 \mu_{T}\right)\left(T_{i, j}^{f+0.5}-T_{i, j-1}^{f+0.5}\right)+2 \mu_{T}\left(T_{i, j}^{f}-T_{i, j-1}^{f}\right)\right] \frac{\left(\Phi_{1}\right)_{i, j}}{\left(R_{1}\right)_{i, j}^{f+0.5}}\right|^{\text {if } j>0} \\
& +\left.\left[-\left(1+2 \mu_{T}\right)\left(T_{i, j}^{f+0.5}-T_{i+1, j}^{f+0.5}\right)+2 \mu_{T}\left(T_{i, j}^{f}-T_{i+1, j}^{f}\right)\right] \frac{\left(\Phi_{2}\right)_{i, j}}{\left(R_{2}\right)_{i, j}^{f+0.5}}\right|^{\text {if } i<n_{r}} \\
& +2 c_{i, j}^{f+0.5} \frac{\left(1+4 \mu_{q}\right) T_{i, j}^{f+0.5}-2 \mu_{q} T_{i, j}^{f}}{\Delta t}+\left(1+2 \mu_{q}\right) Q_{i, j}^{f+1}-2 \mu_{q} Q_{i, j}^{f+0.5}
\end{aligned}
$$

The initial conditions (1.10) are implemented as

$$
T_{i, j}^{0}=T_{0}\left(r_{i}, z_{j}\right) \quad T_{i, j}^{0.5}=T_{i, j}^{0}+0.5 \Delta t v_{0}\left(r_{i}, z_{j}\right)
$$

In a similar way, one can obtain the other variants of ADI, e.g. $(1,3)-(2,4),(2,4)-(1,3)$, etc, by replacing the indexes of directions in the sums in Eqs. (3.18) and (3.19).

Both systems of equations lead to systems with three-diagonal matrices.

\section{Results}

Numerical simulations of the thermal process in a thin film (chromium) subjected to the shortpulse laser heating have been done. Thermophysical parameters of chromium are the following: 
$\lambda=93 \mathrm{~W} /(\mathrm{mK}), c=3.2148 \cdot 10^{6} \mathrm{~J} /\left(\mathrm{m}^{3} \mathrm{~K}\right), \tau_{q}=0.136 \cdot 10^{-12} \mathrm{~s}, \tau_{T}=7.86 \cdot 10^{-12} \mathrm{~s}($ Tang and Araki, 1999). The cylindrical domain with dimensions $Z=100 \cdot 10^{-9} \mathrm{~m}, R=100 \cdot 10^{-9} \mathrm{~m}$ is considered. The parameters of the Gaussian-shaped pulse are equal to: $r_{d}=50 \cdot 10^{-9} \mathrm{~m}$, $I_{0}=13.7 \mathrm{~W} / \mathrm{m}^{2}, R_{f}=0.93, \delta=15.3 \cdot 10^{-12} \mathrm{~m}, t_{p}=100 \cdot 10^{-15} \mathrm{~s}$. The initial temperature and the initial heating rate of the metal are equal to: $T_{0}(r, z)=20^{\circ} \mathrm{C}$ and $v_{0}(r, z)=0 \mathrm{~K} / \mathrm{s}$. Different mesh steps: $\Delta z, \Delta r$ and different time step $\Delta t$ are tested in this example.

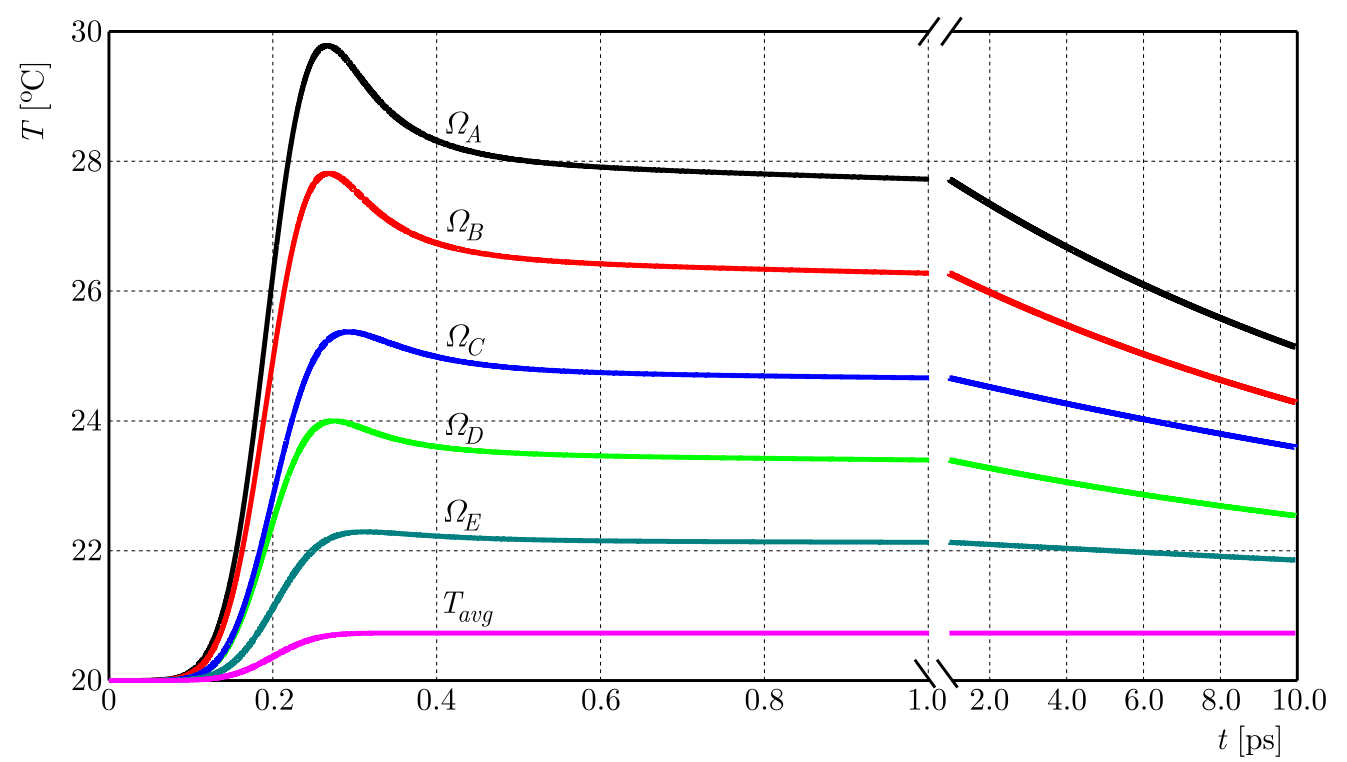

Fig. 4. Heating curves at the selected control volumes $\Omega_{k}$ and the average temperature of the domain

In Fig. 4, the temperature histories (calculated as the average temperature) at five selected control volumes of the domain

$$
\begin{aligned}
& \Omega_{A}=\left\{(r, z) \mid 0 \leqslant r \leqslant \frac{\Delta r}{2}, 0 \leqslant z \leqslant \frac{\Delta z}{2}\right\} \\
& \Omega_{B}=\left\{(r, z) \mid \frac{R}{4}-\frac{\Delta r}{2} \leqslant r \leqslant \frac{R}{4}+\frac{\Delta r}{2}, 0 \leqslant z \leqslant \frac{\Delta z}{2}\right\} \\
& \Omega_{C}=\left\{(r, z) \mid \frac{R}{2}-\frac{\Delta r}{2} \leqslant r \leqslant \frac{R}{2}+\frac{\Delta r}{2}, 0 \leqslant z \leqslant \frac{\Delta z}{2}\right\} \\
& \Omega_{D}=\left\{(r, z) \mid 0 \leqslant r \leqslant \frac{\Delta r}{2}, \frac{Z}{5}-\frac{\Delta z}{2} \leqslant z \leqslant \frac{Z}{5}+\frac{\Delta z}{2}\right\} \\
& \Omega_{E}=\left\{(r, z) \mid \frac{R}{2}-\frac{\Delta r}{2} \leqslant r \leqslant \frac{R}{2}+\frac{\Delta r}{2}, \frac{Z}{5}-\frac{\Delta z}{2} \leqslant z \leqslant \frac{Z}{5}+\frac{\Delta z}{2}\right\}
\end{aligned}
$$

are shown. In this figure, the course of the average temperature $T_{\text {avg }}$ of the whole cylindrical domain is also presented. Here, the calculations are performed using the ADI variant: $(1,2)-(3,4)$ for the following parameters of meshes: $\Delta z=10^{-9} \mathrm{~m}, \Delta r=10^{-9} \mathrm{~m}, \Delta t=10^{-16} \mathrm{~s}$.

The courses of isotherms for the selected moments of time: $0.3,0.5,1$ and 10 ps are presented in Fig. 5.

Next, the comparison of different variants of the ADI method (here: schemas $(1,2)-(3,4)$ and $(1,3)-(2,4)$ are chosen) for different sizes of meshes is studied. Adequate numerical simulations have been performed. The differences in the numerical solutions are hard to see in the graphs. So, the numerical results (as the average temperature at the selected control volumes (4.1) at time $t=0.3 \mathrm{ps}$ ) for different sizes of meshes are collected in Table 1 . The analytical solution of the problem considered is so far unknown in literature and, hence, it is difficult to estimate which numerical scheme is the best. 

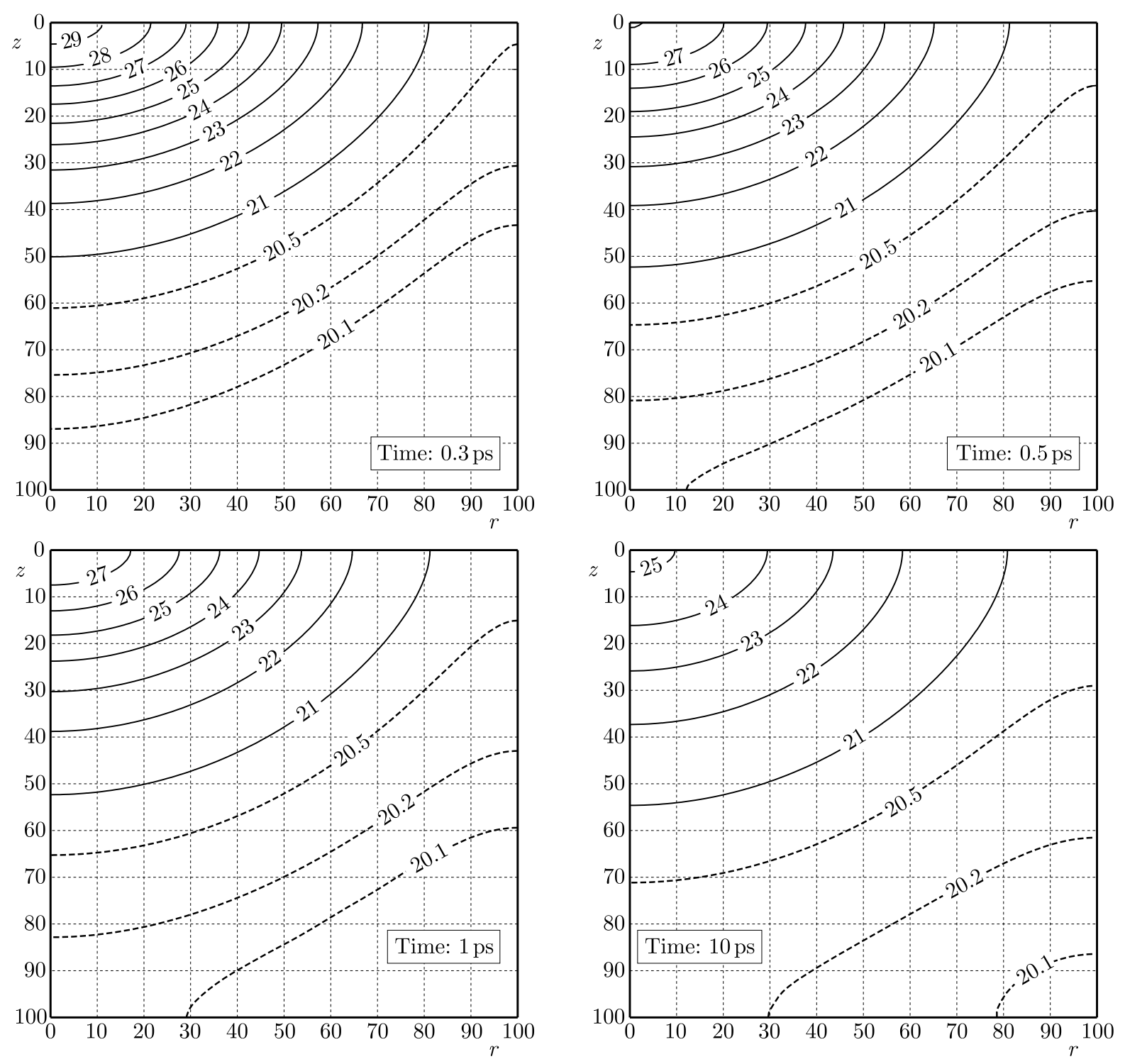

Fig. 5. Courses of isotherms in the cross-section of the domain for different times

Table 1. Numerical results (temperature) for different sizes of meshes

\begin{tabular}{|c|c|c|c|c|c|c|c|}
\hline \multirow{2}{*}{$\begin{array}{c}\Delta r=\Delta z \\
{[\mathrm{~m}]}\end{array}$} & \multirow{2}{*}{$\begin{array}{l}\Delta t \\
{[\mathrm{~s}]}\end{array}$} & \multirow{2}{*}{ Method } & \multicolumn{5}{|c|}{ Average temperature at time $t=0.3 \mathrm{ps}$ at selected $\mathrm{CV}\left[{ }^{\circ} \mathrm{C}\right]$} \\
\hline & & & $\overline{\Omega_{A}}$ & $\Omega_{B}$ & $\overline{\Omega_{C}}$ & $\Omega_{D}$ & $\Omega_{E}$ \\
\hline \multirow{6}{*}{$\begin{array}{l}10^{-8} \\
\left(n_{r}=n_{z}\right. \\
=10)\end{array}$} & \multirow{2}{*}{$10^{-15}$} & $\overline{(1,2)-}$ & 29.407462 & 28.175041 & 23.939626 & 25.296615 & 22.257754 \\
\hline & & $(1,3)-(2,4)$ & 29.407477 & 28.175054 & 23.939633 & 25.296637 & 22.257766 \\
\hline & \multirow{2}{*}{$10^{-16}$} & $(1,2)-(3,4)$ & 29.423528 & 28.188169 & 23.943812 & 25.297708 & 22.256563 \\
\hline & & $(1,3)-(2,4)$ & 29.423528 & 28.188169 & 23.943812 & 25.297709 & 22.256563 \\
\hline & \multirow{2}{*}{$10^{-17}$} & $(1,2)-(3,4)$ & 29.425095 & 28.189446 & 23.944210 & 25.297781 & 22.256427 \\
\hline & & $(1,3)-(2,4)$ & 29.425095 & 28.189446 & 23.944210 & 25.297781 & 22.256427 \\
\hline \multirow{6}{*}{$\begin{array}{l}10^{-9} \\
\left(n_{r}=n_{z}\right. \\
=100\end{array}$} & \multirow{2}{*}{$10^{-15}$} & $(1,2)-(3,4)$ & 29.385557 & 27.542517 & 23.924927 & 25.361719 & 22.284553 \\
\hline & & $(1,3)-(2,4)$ & 29.387539 & 27.544204 & 23.926049 & 25.365099 & 22.286141 \\
\hline & \multirow{2}{*}{$10^{-16}$} & $(1,2)-(3,4)$ & 29.403565 & 27.555886 & 23.930159 & 25.366322 & 22.284954 \\
\hline & & $(1,3)-(2,4)$ & 29.403585 & 27.555903 & 23.930170 & 25.366356 & 22.284970 \\
\hline & \multirow{2}{*}{$10^{-17}$} & $(1,2)-(3,4)$ & 29.405160 & 27.557048 & 23.930567 & 25.366453 & 22.284839 \\
\hline & & $(1,3)-(2,4)$ & 29.405159 & 27.557046 & 23.930567 & 25.366452 & 22.284839 \\
\hline
\end{tabular}


The total energy $(\Delta Q[\mathrm{~J}])$ applied to the considered domain during one laser pulse is equal to

$$
\begin{aligned}
\Delta Q & =\int_{0}^{4 t_{p}} \int_{0}^{Z} \int_{0}^{R} \int_{0}^{2 \pi} Q(r, z, t) r d \Phi d r d z d t \\
& =I_{0}\left(1-R_{f}\right) \pi \frac{r_{d}^{2}}{2}\left[1-\exp \left(-\frac{R^{2}}{r_{d}^{2}}\right)\right]\left[1-\exp \left(-\frac{Z}{\delta}\right)\right][\operatorname{erf}(2 \beta)-\operatorname{erf}(-2 \beta)]
\end{aligned}
$$

The laser energy causes a rise in temperature equal to $\Delta T\left[{ }^{\circ} \mathrm{C}\right]$ in the domain (assuming adiabatic conditions at all boundaries)

$$
\Delta T=\frac{\Delta Q}{c V}
$$

where $V=\pi R^{2} Z\left[\mathrm{~m}^{3}\right]$ is the volume of the whole domain. For the above mentioned values of parameters, the temperature increases by $\Delta T=0.731048^{\circ} \mathrm{C}$ after one laser pulse. This value is used, among others, to compare the correctness of the numerical results. It should be pointed out that the analytical value and numerical values are practically the same (the errors are of the order $\left.0.00001^{\circ} \mathrm{C}\right)$.

\section{Conclusion}

The dual phase lag model seems to be adequate for mathematical description of microscale heat transfer. In many situations when analytical solutions are not known, the numerical solutions are desired to be found. To obtain an effective solution to the considered problem, the algorithm based on the control volume method is presented. The DPLM consists of the partial differential equation of a hyperbolic type, and thus the more complex numerical schemes should be developed, of course. In this paper, the Alternating Direction Implicit scheme is constructed. This scheme can be easily implemented in computer programs. In opposite to other schemes, such as the Crank-Nicolson scheme, where it is necessary to solve the system of equations characterized by a 5-band matrix, the ADI method requires double solving of the systems with 3-band matrices. From the computational point of view, the solution of the system of equations with the 3-band matrix is efficient and fast. The numerical scheme allows one to use the thermophysical parameters of the material (i.e. thermal conductivity and volumetric specific heat) as the temperature-dependent (in this paper these parameters are assumed to be constant values). The results (see: the temperatures presented in Table 1) obtained by application of the two types of numerical schemes are very similar, especially the results obtained for the same set of the parameters of meshes: $\{\Delta z, \Delta r, \Delta t\}$ are practically identical.

\section{Acknowledgement}

The paper is a part of research project 2015/19/B/ST8/01101 sponsored by NSC (Poland).

\section{References}

1. Belkhayat-Piasecka A., Korczak A., 2016, Modeling of transient heat transport in metal films using the interval lattice Boltzmann method, Bulletin of the Polish Academy of Sciences Technical Sciences, 64, 3, 599-505

2. Cattaneo C., 1958, A form of heat conduction equation which eliminates the paradox of instantaneous propagation, Compte Rendus, 27, 431-433 
3. Chen G., Borca-Tasciuc D., YAng R.G., 2004, Nanoscale heat transfer, [In:] Encyclopedia of Nanoscience and Nanotechnology, Nalwa H.S. (Edit.), Vol. X, 1-30, http://www.aspbs.com/enn.html

4. Chen J.K., Beraun J.E., 2001, Numerical study of ultrashort laser pulse interactions with metal films, Numerical Heat Transfer, Part A, 40, 1-20

5. Chen W.H., Cheng H.C., Hsu Y.C., 2007, Mechanical properties of carbon nanotubes using molecular dynamics simulations with the inlayer van der Waals interactions, CMES: Computer Modeling in Engineering and Sciences, 20, 2, 123-145

6. Ciesielski M., Duda M., Mochnacki B., 2016, Comparison of bio-heat transfer numerical models based on the Pennes and Cattaneo-Vernotte equations, Journal of Applied Mathematics and Computational Mechanics, 15, 4, 33-38

7. Ciesielski M., Mochnacki B., 2014, Application of the control volume method using the Voronoi polygons for numerical modeling of bio-heat transfer processes, Journal of Theoretical and Applied Mechanics, 52, 4, 927-935

8. Dai W., NAssar R., 2000, A domain decomposition method for solving three-dimensional heat transport equations in a double-layered thin film with microscale thickness. Numerical Heat Transfer, Part A, 38, 243-255

9. Dziatkiewicz J., Kuś W., Majchrzak E., Burczyński T., Turchan L., 2014, Bioinspired identification of parameters in microscale heat transfer, International Journal for Multiscale Computational Engineering, 12, 1, 79-89

10. Escobar R.A., Ghai S.S., Jhon M.S., Amon C.H., 2006, Multi-length and time scale thermal transport using the lattice Boltzmann method with application to electronics cooling, International Journal of Heat and Mass Transfer, 49, 97-107

11. KABA I.K., DAI W., 2005, A stable three-level finite difference scheme for solving the parabolic two-step model in a 3D micro-sphere heated by ultrashort-pulsed lasers, Journal of Computational and Applied Mathematics, 181, 125-147

12. Lin Z., Zhigilei L.V., 2008, Electron-phonon coupling and electron heat capacity of metals under conditions of strong electron-phonon nonequilibrium, Physical Review, B, 77, 075133-1-075133-17

13. Liu D.S., Tsai C.Y., 2009, Estimation of thermo-elasto-plastic properties of thin-film mechanical properties using MD nanoindentation simulations and an inverse FEM/ANN computational scheme, CMES: Computer Modeling in Engineering and Sciences, 39, 1, 29-47

14. Majchrzak E., 2012, Parabolic and hyperbolic two-temperature models of microscopic heat transfer. Comparison of numerical solutions, Materials Science Forum, 706-709, 1454-1459

15. Majchrzak E., Dziatkiewicz J., 2015, Analysis of ultashort laser pulse interactions with metal films using a two-temperature model, Journal of Applied Mathematics and Computational Mechanics, 14, 2, 31-39

16. Majchrzak E., Mochnacki B., 2014, Sensitivity analysis of transient temperature field in microdomains with respect to the dual phase lag model parameters, International Journal for Multiscale Computational Engineering, 12, 1, 65-77

17. Majchrzak E., Mochnacki B., Greer A.L., Suchy J.S., 2009a, Numerical modeling of short pulse laser interactions with multi-layered thin metal films, CMES: Computer Modeling in Engineering and Sciences, 41, 2, 131-146

18. Majchrzak E., Mochnacki B., Suchy J.S., 2009b, Numerical simulation of thermal processes proceeding in a multi-layered film subjected to ultrafast laser heating, Journal of Theoretical and Applied Mechanics, 47, 2, 383-396

19. Majchrzak E., Turchan L., 2016, Modeling of phase changes in the metal micro-domains subjected to ultrafast laser heating using dual-phase lag equation, Materialwissenschaft und Werkstofftechnik, 47, 5/6, 409-418 
20. Mitra K., Kumar S., Vedavarz A., Moallemi M.K., 1995, Experimental evidence of hyperbolic heat conduction in processed meat, ASME Journal of Heat Transfer, 17, 568-573

21. Mochnacki B., Ciesielski M., 2012, Numerical model of thermal processes in domain of thin film subjected to a cyclic external heat flux, Materials Science Forum, 706-709, 1460-1465

22. Mochnacki B., Ciesielski M., 2015, Micro-scale heat transfer. Algorithm basing on the control volume method and the identification of relaxation and thermalization times using the search method, Computer Methods in Materials Science, 15, 2, 353-361

23. Mochnacki B., Paruch M., 2013, Estimation of relaxation and thermalization times in microscale heat transfer, Journal of Theoretical and Applied Mechanics, 51, 4, 837-845

24. Orlande H.R.B., ÖzIşıK M.N., Tzou D.Y., 1995, Inverse analysis for estimating the electronphonon coupling factor in thin metal films, Journal of Applied Physics, 78, 3, 1843-1849

25. Smith A.N., Norris P.M., 2003, Microscale Heat Transfer, Chapter 18 in: Heat Transfer Handbook, John Wiley \& Sons

26. TAng D.W., Araki N., 1999, Wavy, wavelike, diffusive thermal responses of finite rigid slabs to high-speed heating of laser-pulses, International Journal of Heat and Mass Transfer, 42, 855-860

27. Theodosiou T.C., Saravanos D.A., 2007, Molecular mechanics based finite element for carbon nanotube modeling, CMES: Computer Modeling in Engineering and Sciences, 19, 121-134

28. Tian W., Yang R., 2008, Phonon transport and thermal conductivity percolation in random nanoparticle composites, CMES: Computer Modeling in Engineering and Sciences, 24, 123-142

29. Tzou D.Y., 2015, Macro- to Microscale Heat Transfer: The Lagging Behavior, John Wiley \& Sons, Ltd.

30. Zhang Z.M., 2007, Nano/microscale Heat Transfer, McGraw-Hill, New York 